\title{
Sufficient conditions for a continuous linear operator to be weakly compact
}

\section{Joe Howard and Kenneth Melendez}

\begin{abstract}
A locally convex topological vector (LCTV) space $E$ is said to have property $V$ (Dieudonné property) if for every complete separated LCTV space $F$, every unconditionally converging (weakly completely continuous) operator $T: E \rightarrow F$ is weakly compact. First, an investigation of the permanence of property $V$ is given. The permanence of the Dieudonne is analogous. Relationships between property $V$ and the Dieudonné property are then given.
\end{abstract}

1. Preliminaries

In the following definitions $(E, \tau)$ and $\left(F, \tau^{\prime}\right)$ will denote separated locally convex topological vector spaces (LCTVS) with topologies $\tau$ and $\tau^{\prime}$ respectively. All linear operators are to be continuous. We use this fact without making further reference to it. A series $\sum_{i=1}^{\infty} x_{i}$ in $(E, \tau)$ is unconditionally convergent (uc) if it is subseries convergent relative to $\tau$. Equivalent conditions for uc series are given in [4]. A series $\sum_{i=1}^{\infty} x_{i}$ in $(E, \tau)$ is said to be weakly unconditionally convergent (wuc) if $\sum_{i=1}^{\infty}\left|f\left(x_{i}\right)\right|<\infty$ for every $f$ in $E^{\prime}$. An equivalent condition for wuc is that $S=\left\{\sum_{i \in \sigma} x_{i}: \sigma\right.$ finite $\}$ be bounded relative to $\tau$. 
A linear operator $T: E \rightarrow F \quad(F$ complete $)$ is said to be unconditionally converging (uc operator) if it sends every wuc series in $E$ into uc series in $F, T$ is said to be weakly compact if $T$ maps bounded sets of $E$ into weakly relatively compact sets of $F$. This definition, which can be found in Lemma 1 of [2], differs slightly from the more common one given in [1]. It is easy to show using condition (E) of [4] that every weakly compact operator is a uc operator.

$N(E)$ is to denote $J E$ ( $J$ is the natural map) plus all $\sigma\left(E^{\prime \prime}, E^{\prime}\right)$ limits of wuc series in $E, N(E)$ is a subset of $E^{\prime \prime}$. The following theorem is a consequence of Proposition 9.4 .9 of [1], and therefore a proof will not be given.

THEOREM 1.1. The following conditions on $E$ are equivalent.

(1) For every complete separated LCTVS $F$, every uc operator $T: E \rightarrow F$ is weakly compact.

(1') As (1), F being restricted to a Banach space.

(2) Any continuous linear mop $T: E \rightarrow F$ ( $F$ as in (1)) for which $T^{\prime \prime}(N(E)) \subset F \quad$ satisfies $T^{\prime \prime}\left(E^{\prime \prime}\right) \subset F$.

(2') As (2), F being restricted to a Bonach space.

(3) Any equicontinuous, convex, balanced, and $\sigma\left(E^{\prime}, N(E)\right)$-compact set in $E^{\prime}$ is also $\sigma\left(E^{\prime}, E^{\prime \prime}\right)$-compact.

That (1) and (2) are equivalent can be seen from the facts that $T: E \rightarrow F$ is uc if and only if $T^{\prime \prime}(N(E)) \subset F$ and $T: E \rightarrow F$ is weakly compact if and only if $T^{\prime \prime}\left(E^{\prime \prime}\right) \subset F$.

DEFINITION 1.2. Any LCTVS $E$ which has one of the above equivalent properties is said to have property $V$.

This definition is a generalization of property $V$ for Banach spaces studied by Petczyński in [5].

Let $K(E)$ denote $J E$ plus all $\sigma\left(E^{\prime \prime}, E^{\prime}\right)$-limits of weak Cauchy sequences in $E$. By using $K(E)$ instead of $N(E)$ in Proposition 9.4.9 of [1], we have a theorem similar to Theorem 1.1 above. We state this as a definition.

DEFINITION 1.3. $E$ is said to have the Dieudonné property if one of 
the following equivalent properties is satisfied.

(I) For every complete separated LCTVS $F$, every operator $T: E \rightarrow F$ which transforms weak Cauchy sequences into weakly convergent sequences is weakly compact.

(1') As (1), $F$ being restricted to a Banach space.

(2) Any continuous linear map $T: E \rightarrow F$ ( $F$ as in (1)), for which $T^{\prime \prime}(K(E)) \subset F$ satisfies $T^{\prime \prime}\left(E^{\prime \prime}\right) \subset F$.

(2') As (2), F being restricted to a Banach space.

(3) Any equicontinuous, convex, balanced and $\sigma\left(E^{\prime}, K(E)\right)$-compact set in $E^{\prime}$ is also $\sigma\left(E^{\prime}, E^{\prime \prime}\right)$-compact.

A complete discussion of the Dieudonne property is found in [1] and [2].

Another property which is somewhat related to both property $V$ and the Dieudonne property is the following property.

DEFINITION 1.4. A LCTV space $E$ is said to have property (u) if for every weak Cauchy sequence $\left\{x_{n}\right\}$ in $E$ there exists a wuc series $\sum u_{k}$ such that the sequence $\left\{x_{n}-\sum_{k=1}^{n} u_{k}\right\}$ converges weakly to 0 .

\section{Permanence of property $V$}

Since condition (3) of Theorem 1.1 is a condition on the dual space $E^{\prime}$, compatible topologies for $E$ must all agree on $E$ having (or not having) property $V$. Hence, if $E$ is a Banach space having (not having) property $V$, then $E$ with the weak topology has (does not have) property $V$. In particular, $Z_{1}$ with the weak topology does not have property $V$ since $l_{I}$ with the norm topology does not [5]. An example of a LCTV space which does have property $V$ is a reflexive space.

PROPOSITION 2.1. Suppose $E$ is the regular inductive lirrit [3] of the LCTVS $E_{n}$. If each $E_{n}$ has property $V$, then $E$ has property $V$.

Proof. Let $T: E \rightarrow F$ be a uc operator, $F$ complete, and $B$ a 
bounded subset of $E$. Then for some $n, f_{n}^{-1}(B)$ is a bounded set in $E_{n}$ where $f_{n}$ is the continuous linear mapping from $E_{n}$ to $E$ such that $\cup f_{n}\left(E_{n}\right)$ spans $E$. Now define $T_{n}$ such that the diagram

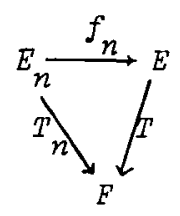

is commutative. Then $T_{n}=T f_{n}$ is a uc operator and since $E_{n}$ has property $V, T_{n}$ is a weakly compact operator. Hence the weak closure of $T_{n}\left(f_{n}^{-1}(B)\right\}=T(B)$ is compact in the weak topology of $F$. Therefore $T$ is weakly compact, and $E$ has property $V$.

EXAMPLE 2.2. Projective limits do not necessarily preserve property V.

Proof. Let $R$ denote the reals and define the map $h: E \rightarrow E_{f}=R$ by $h: e \rightarrow f(e)$ where $f$ belongs to $E^{\prime}$. Now if we take $E=\tau_{1}$ with the weak topology, then $l_{1}$ is the projective limit of $\left|z_{\infty}\right|$ copies of $R$, where $\left|z_{\infty}\right|$ denotes the cardinality of $z_{\infty} \cdot R=E_{f}$ has property $V$ since it is reflexive, but $l_{1}$ with the weak topology does not.

REMARK. Suppose $E=E_{1} \times E_{2}$. Then $T: E+F$ is weakly compact if and only if $T \mid E_{1}$ and $T \mid E_{2}$ (the restriction of $T$ to $E_{1}$ and $E_{2}$, respectively) is weakly compact. This is also true for uc operators. Hence, if $E_{1}, E_{2}, \ldots, E_{n}$ are ICTVS with property $V$, then $E_{1} \times E_{2} \times \ldots \times E_{n}$ has property $V$. The following proposition shows that this is also true for infinite products.

PROPOSITION 2.3. Suppose $E$ is the infinite direct product of the LCTVS $E_{n}$. If each $E_{n}$ has property $V$, then $E$ has property $V$.

Proof. Let $T: E \rightarrow F$ be a continuous operator, $F$ a Banach space, 
and $h_{n}$ the natural map of $E_{n}$ into $E$. Then $T_{n}=T \circ h_{n}$ is continuous from $E_{n}$ into $F$, so $T_{n}=0$ for all but a finite set of indices. Therefore, it suffices to prove the case for a finite product. But this is contained in the above remark.

REMARK. The direct sum of spaces with property $V$ has property $V$. The proof is analogous to that for the direct products.

PROPOSITION 2.4. If $E$ is a normed linear space having property $V$, then every quotient space $E$ has property $V$.

Proof. Let $M$ be a subspace of $E$. Define $T$ such that the di agram

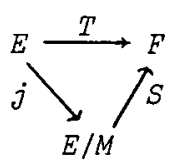

is commutative where $j$ is the canonical map and $F$ is complete. Assume $S$ is a uc operator and let $\sum x_{n}$ be a wuc series in $E$. Since $j$ is continuous, $\sum j\left(x_{n}\right)$ is a wuc series in $E / M$ and therefore $\sum T\left(x_{n}\right)=\sum S j\left(x_{n}\right)$ is a uc series in $F$. So $T$ is a uc operator and since $E$ has property $V, T$ is a weakly compact operator.

Let $B \neq\{0\}$ be a bounded set in $E / M$. Since $E$ is a normed space, $j^{-1}(B)$ is a bounded set in $E$ and hence the weak closure of $T j^{-1}(B)=S(B)$ is compact in the weak topology of $F$. Therefore $S$ is a weakly compact operator, so $E / M$ has property $V$.

REiviARKS. (1) For LCTVS, Property $V$ is not necessarily preserved for quotient spaces. In Problem 20, page 195 of [7], there is given a Montel space $E$ which has a quotient space isomorphic to $\tau_{1}$. Since $E$ is a Montel space, $E$ is reflexive and hence has property $V$. However, $I_{I}$ does not have property $V$. Since an inductive limit topology can be considered as a quotient topology, this example also shows that property $V$ is not preserved by inductive limits. 
(2) Property $V$ is not preserved for subspaces since $l_{1}$ is linearly isometric to a subspace of $C(S), S$ a compact Hausdorff space, and $C(S)$ has property $V$ [5] while $l_{1}$ with the norm topology does not. However a space $E$ has property $V$ if and only if every complemented subspace has property $V$.

(3) It is an open question whether property $V$ is preserved under tensor products. Swartz has partially answered this question in [8].

\section{The Dieudonne property and property $\boldsymbol{V}$}

Permanence properties for the Dieudonné property are analogous to those for property $V$; hence they are omitted.

If $E$ has property $V$ then $E$ has the Dieudonné property since every wcc operator (a wcc. operator transforms weak Cauchy ssquences into weakly convergent sequences) is a uc operator. In general the converse is not true (Example 3.3), however a space having property (u) (Definition 1.4) is a sufficient condition for the converse to hold.

LEMMA 3.1. $E$ has property (u) if and only if $N(E)=K(E)$.

Proof. Since $N(E) \subset K(E)$ it will suffice to show $K(E) \subset N(E)$. If $G \in K(E)$, then there exists a weak Cauchy sequence $\left\{x_{n}\right\}$ in $E$ such that $w^{*}-\lim _{n} J x_{n}=G$. Since $E$ has property $(u)$, there exists a wuc series $\sum u_{i}$ in $E$ such that $\left\{x_{n}-\sum_{i=1}^{n} u_{i}\right\}$ converges weakly to 0 , thus $w^{*}-\lim _{n} \sum_{i=1}^{n_{n}} J u_{i}=G$ and $G \in N(E)$.

Conversely, assume $K(E)=N(E)$ and let $\left\{x_{n}\right\}$ be a weak Cauchy sequence in $E$. Since $\left\{x_{n}\right\}$ is weak Cauchy there exists a $G \in K(E)$ such that $w^{*}-\lim _{n} J x_{n}=G$, and since $K(E)=N(E)$ we have $G \in N(E)$, which implies that there exists a wuc series $\sum u_{i}$ such that 
$w^{*}-\lim \sum_{i=1}^{n} J u_{i}=G$, thus $\left\{x_{n}-\sum_{i=1}^{n} u_{i}\right\}$ converges weakly to 0 , so $E$ has property $(u)$.

THEOREM 3.2. If $E$ has property $(u)$, then $E$ has property $V$ if and only if $E$ has the Dieudonne property.

Proof. It suffices to show that every uc operator is a wcc operator. Let $T: E \rightarrow E$ be a uc operator. Then $T^{\prime \prime}(N(E)) \subset \cdot J E$, but since $E$ has property $(u), N(E)=K(E)$, so $T^{\prime \prime}(K(E)) \subset J F$. Since $T$ is a wec operator if and only if $T^{\prime \prime}(K(E)) \subset J F, T$ is a wcc operator.

REMARK. It is not possible to refine Theorem 3.2 to: $E$ has property $V$ if and only if $E$ has the Dieudonné property and property $(u)$. For example $C[0,1]$ has both property $V$ and the Dieudonne property, but not property $(u)$.

EXAMPLE 3.3. James defined a Banach space $B_{3}$ such that $B_{3}, B_{3}^{\prime}$, and $B_{3}^{: \prime}$ are separable but $B_{3}^{\prime \prime \prime}$ is non-separable and $B_{3}^{\prime \prime}=B_{3} \oplus l_{1} \cdot B_{3}^{\prime \prime}$ is separable, so every bounded sequence in $B_{3}$ will have a Cauchy subsequence, and thus every wcc operator will be weakly compact. Hence $B_{3}^{\prime}$ has the Dieudonné property.

However, the identity map $i: B_{3}^{\prime} \rightarrow B_{3}^{\prime}$ is a uc operator, since if $B_{3}^{\prime}$ contained a subspace isomorphic to $c_{0}$, it would contain a subspace isomorphic to $m$ and $B_{3}^{\prime}$ would not be separable, a contradiction. If $i$ were weakly compact, then the unit disk of $B_{3}^{\prime}$ would be weakly compact, hence $B_{3}^{\prime}$ would be reflexive, which it is not, so $B_{3}^{\prime}$ does not have property $V$. Notice $i$ is an example of a uc operator that is not a wec operator.

REMARK. Several conclusions can easily be seen by considering the sets $N(X)$ and $K(X)$. Here are a few.

(1) If $N(X)=X^{\prime \prime}$, then $X$ has property $V$.

(2) If $K(X)=X^{\prime \prime}$, then $X$ has the Dieudonné property.

(3) $N(X)=J X$ if and only if $X$ has no subspace isomorphic to $c_{0}$ 
(equivalent to every wuc series is $u c$ ).

(4) $X$ is weakly complete if and only if $X$ has property (u) and $N(X)=J X$ if and only if $K(X)=J X$.

It can be shown that if $Y$ is a subspace of $X$, then $N(Y)=Y^{\prime \prime} \cap N(X)$ and $K(Y)=Y^{\prime \prime} \cap K(X)$. This gives the inheritance properties (such as those given in this paper) for subspaces.

\section{References}

[1] R.E. Edwards, Functional analysis. Theory and applications (Holt, Rinehart and Winston, New York, Chicago, San Francisco, Toronto, London, 1965).

[2] A. Grothendieck, "Sur les applications linéaires faiblement compactes d'espaces du type $C(K)$ ", Canad. J. Math. 5 (1953), 129-173.

[3] L.V. Kantorovich and G.P. Akilov, Functional analysis in normed spaces (translated from the Russian by D.E. Brown; The Macmillan Company, New York; Pergamon Press, Oxford, London, Edinburgh, New York, Paris, Frankfurt, 1964).

[4] Charles W. McArthur, "On a theorem of Orlicz and Pettis", Pacific J. Math. 22 (1967), 297-302.

[5] A. Petczyński, "Banach spaces on which every unconditionally converging operator is weakly compact", Buzl. Acad. Polon. Sci. Sér. Sci. math. astronom. phys. 10 (1962), 641-648.

[6] A.P. Robertson and Wendy Robertson, Topological vector spaces (Cambridge Tracts in Mathematics and Mathematical Physics, 53. (Cambridge University Press, Cambridge, 1964).

[7] Helmut H. Schaefer, Topological vector spaces (The Macmillan Company, New York; Collier-Macmillan, London, 1966).

[8] C. Swartz, "Unconditionally converging operators on the space of continuous functions", (to appear).

Department of Mathematics and Statistics,

Oklahoma State University,

Still iwater,

Oklahoma, USA. 